\title{
HE4 Serum Levels in Patients with BRCA1 Gene Mutation Undergoing Prophylactic Surgery as well as in Other Benign and Malignant Gynecological Diseases
}

\author{
Anita Chudecka-Głaz, Aneta Cymbaluk-Płoska, Aleksandra Strojna, and Janusz Menkiszak \\ Department of Gynecological Surgery and Gynecological Oncology of Adults and Adolescents, \\ Pomeranian Medical University, Szczecin, Poland \\ Correspondence should be addressed to Anita Chudecka-Głaz; anitagl@poczta.onet.pl
}

Received 4 September 2016; Revised 17 November 2016; Accepted 1 December 2016; Published 15 January 2017

Academic Editor: Anja Hviid Simonsen

Copyright (C) 2017 Anita Chudecka-Głaz et al. This is an open access article distributed under the Creative Commons Attribution License, which permits unrestricted use, distribution, and reproduction in any medium, provided the original work is properly cited.

\begin{abstract}
Objective. We assess the behavior of serum concentrations of HE4 marker in female carriers of BRCA1 and assess the diagnostic usefulness of HE4 in ovarian and endometrial cancer. Methods. A total of 619 women with BRCA1 gene mutation, ovarian, endometrial, metastatic, other gynecological cancers, or benign gynecological diseases were included. Intergroup comparative analyses were carried out, the BRCA1 gene carriers subgroup was subjected to detailed analysis, and ROC curves were determined for the assessment of diagnostic usefulness of HE4 in ovarian and endometrial cancer. Results. Statistically lower serum HE4 and CA 125 levels were observed in BRCA1 gene mutation premenopausal carriers. Occult ovarian/fallopian tube cancer was found 3.6\%. Each of those patients was characterized by slightly elevated levels of either CA 125 (63.9 and $39.4 \mathrm{U} / \mathrm{mL})$ or HE4 (79 pmol/L). The ROC-AUC curves were 0.892 and 0.894 for diagnostic usefulness of ovarian cancer and 0.865 for differentiation of endometrial cancer from endometrial polyps. Conclusions. Patients with BRCA1 gene mutations have relatively low serum HE4 levels. Even the slightest elevation in HE4 or CA 125 levels in female BRCA1 carriers undergoing prophylactic surgery should significantly increase oncological alertness. The HE4 marker is valuable in ovarian and uterine cancer diagnosis.
\end{abstract}

\section{Introduction}

In recent years hundreds of proteins have been tested for their importance as markers in cancer diseases. A large part of these studies consisted of experiments involving new markers of ovarian cancer. Despite the use of novel imaging techniques as well as increasingly advanced therapeutic methods, ovarian cancer continues to pose the greatest challenge in gynecological oncology due to the late diagnosis and poor prognosis. As recently as several years ago, the only marker used in clinical practice in ovarian cancer patients was CA125. Only after the studies conducted by Hellström et al. [1] in 2003 and subsequently followed by other authors [2-8], the era of research on a very promising glycoprotein of the fourdisulfide core family has begun. The four-disulfide core family is a heterogeneous group of small, acidic, and thermally stable proteins of varied functionalities. Starting from the early 1990s to date, a total of about 200 articles have been published on the use of human epididymis protein 4 (HE4) in ovarian cancer while nearly 4000 articles on the use of CA125 marker have been published to date since the early 1980s. It is therefore clear that further research on HE4 is required before its usefulness raises no scientific doubts. As of today, it appears that HE4 is a sensitive and, first of all, specific marker of malignant epithelial ovarian cancers $[9,10]$. Combined with CA125, HE4 offers a useful diagnostic method as a part of ROMA algorithm for prediction of the malignant nature of an ovarian tumor $[8,11,12]$. The marker may be successfully used in the monitoring of ovarian cancer [13]; studies of recent years also suggest a high prognostic potential of HE4 [14]. No screening tests have been developed for ovarian cancer to date. The results of all research trials were negative [15]. The only isolated population subgroup that might benefit from ovarian cancer screening tests is patients with mutations within the BRCA1 and BRCA2 genes and burdened by a 
family history of ovarian cancer $[16,17]$. The behavior of the HE4 marker as well as its usefulness in this group of patients has not been unambiguously determined.

\section{Materials and Methods}

A total of 619 patients, 298 premenopausal and 321 postmenopausal, were included in the study in the period from 2010 to 2014 . The age of patients ranged from 18 to 92 . The initial study population consisted of BRCA1 gene mutation carriers presenting at the Department of Gynecological Surgery and Gynecological Oncology of Adults and Adolescents for prophylactic bilateral salpingooophorectomy and female patients with the most common pathologies of the genital organ (gynecological tumors, noncancerous ovarian cysts, uterine myomas, adnexitis, and metastatic ovarian tumors). Patients with a history of renal and lung diseases were not included in the study. The study was approved by Ethics Committee of Pomeranian Medical University and all patients signed the informed consent for participation. After the consent, blood was drawn from the patients and subsequently delivered to the central laboratory, where separation of serum and determination of HE4 and CA125 serum levels were performed. All assessments were made immediately, without the need for freezing of the material.

Table 1 presents the characteristics of the study population. The final distribution of patients into individual groups was performed after histopathology test results were available and after a number of patients were excluded from the study due to elevated serum creatinine levels. Besides the primary distribution of patients into 9 study groups presented in Table 1, the following subgroups were identified:

(i) in group C:
(a) patients with endometrial ovarian cysts
(b) patients with teratoma tumors
(c) patients with hemorrhagic cysts
(d) patients with paraovarian lesions

(ii) in group E:
(a) serous tumors
(b) mucinous tumors
(c) cystadenofibroma

(iii) in group G:
(a) ovarian gonadal tumors
(b) vulvar cancers
(c) cervical cancers

(iv) in group R:
(a) serous cancers
(b) mucinous cancers
(c) endometrial cancers
(d) clear-cell cancers

TABle 1: Patient (groups) demographics.

\begin{tabular}{|c|c|c|c|}
\hline & $n$ & Age mean & Age range \\
\hline All groups & 619 & 51.09 & $18-92$ \\
\hline \multicolumn{4}{|c|}{ Group A: BRCA 1 mutation } \\
\hline All & 83 & 47.9 & $34-64$ \\
\hline Premenopausal & 53 & 43.35 & $34-51$ \\
\hline Postmenopausal & 30 & 56.93 & $48-64$ \\
\hline \multicolumn{4}{|c|}{ Group B: myomas } \\
\hline All & 90 & 47.10 & $25-79$ \\
\hline Premenopausal & 63 & 42.75 & $25-52$ \\
\hline Postmenopausal & 27 & 57.26 & $44-79$ \\
\hline \multicolumn{4}{|c|}{ Group C: nonneoplastic ovarian cysts } \\
\hline All & 82 & 35.4 & $18-75$ \\
\hline Premenopausal & 66 & 30.39 & $18-53$ \\
\hline Postmenopausal & 16 & 56.06 & $54-75$ \\
\hline \multicolumn{4}{|c|}{ Group D: inflammatory diseases } \\
\hline All & 9 & 34.67 & $18-43$ \\
\hline Premenopausal & 9 & 34.67 & $18-43$ \\
\hline Postmenopausal & - & - & - \\
\hline \multicolumn{4}{|c|}{ Group E: benign epithelial ovarian tumors } \\
\hline All & 35 & 48.77 & $18-88$ \\
\hline Premenopausal & 15 & 29.27 & $18-48$ \\
\hline Postmenopausal & 20 & 63.4 & $54-88$ \\
\hline \multicolumn{4}{|c|}{ Group F: endometrial cancers } \\
\hline All & 55 & 66.7 & $54-92$ \\
\hline Premenopausal & 2 & 43.5 & $43-44$ \\
\hline Postmenopausal & 53 & 68.3 & $54-92$ \\
\hline \multicolumn{4}{|c|}{ Group G: other gynecological cancers } \\
\hline All & 38 & 53.84 & $19-92$ \\
\hline Premenopausal & 17 & 37.41 & $19-51$ \\
\hline Postmenopausal & 21 & 67.14 & $52-92$ \\
\hline \multicolumn{4}{|c|}{ Group H: endometrial polyps } \\
\hline All & 95 & 52.92 & $19-83$ \\
\hline Premenopausal & 40 & 39.5 & $19-50$ \\
\hline Postmenopausal & 55 & 62.67 & $49-83$ \\
\hline \multicolumn{4}{|c|}{ Group M: other cancers (metastatic ovarian tumors) } \\
\hline All & 8 & 62.38 & $51-78$ \\
\hline Premenopausal & - & - & - \\
\hline Postmenopausal & 8 & 62.38 & $51-78$ \\
\hline \multicolumn{4}{|c|}{ Group O: ovarian cancers } \\
\hline All & 124 & 58.23 & $30-90$ \\
\hline Premenopausal & 33 & 44.64 & $30-65$ \\
\hline Postmenopausal & 91 & 63.2 & $48-90$ \\
\hline
\end{tabular}

Mean HE4 values were compared in individual groups and subgroups, and relationships between HE4 and CA125 levels were analyzed in the study population. Diagnostic usefulness of HE4 was also compared to that of CA125 in ovarian cancer patients relative to female patients with benign 
ovarian lesions; in addition, diagnostic usefulness of HE4 was compared to that of CA125 in endometrial cancer relative to patients with benign endometrial lesions. All the analyses were carried out in three variants: regardless of hormonal status, in postmenopausal patients and in premenopausal patients. Patients were classified as postmenopausal when the last menstruation occurred more than 12 months ago or if FSH serum levels exceeded $30 \mathrm{U} / \mathrm{L}$.

Incidence of occult ovarian cancers (patients with latent ovarian/fallopian tube cancers were excluded from comparative analysis) and the incidence of breast cancer were analyzed and follow-up examinations were carried out in the group of BRCA1 mutation carriers so as to record new cases of breast cancer or peritoneal cancer. The minimum follow-up period was 1 year.

2.1. Marker Analysis. Assays were performed at the Central Laboratory of the Independent Public Hospital.

CA125 was determined with the Architect i2000 assay from Abbott Diagnostics. The normal range was 1-35 U/mL. Serum HE4 concentrations were measured with the Elecsys ECLIA assay from Roche running on the cobas e 601 analyzer. The measurement range was 15.0-1500 pmol/L. Samples exceeding the upper range were diluted with Elecsys Diluent Multiassay. Manufacturer's instructions were followed and control samples were within the normal range. The normal upper limit range for serum was below $70 \mathrm{pmol} / \mathrm{L}$.

2.2. Statistical Analysis. The statistical analysis was performed using STATISTICA 9.1 PL program.

The descriptive characteristic of the examined population of patients was prepared, determining minimum, maximum mean, and median values. Also the scatter diagrams of empirical values of markers were plotted, subdivided into the studied groups. The mean/median values in particular groups and subgroups were compared using the nonparametric $U$-test of Mann-Whitney.

In order to determine the relation between the analyzed markers, Pearson's linear correlation coefficients were counted and the linear regression function was estimated. For the selected groups the receiver operating characteristic (ROC) curves were obtained and the area under curve (AUC) was calculated with $95 \%$ confidence intervals according to the nonparametric method of DeLong. A $P$ value of $<0.05$ was considered as statistically significant.

\section{Results}

When analyzing all patients regardless of their hormonal status, mean serum HE4 levels in BRCA1 carriers were significantly lower than in the remaining groups (uterine myomas $P=0.0138$; noncancer ovarian cysts $P=0.0001$; adnexitis $P=0.0079$; endometrial cancer $P=0.0000$; other gynecological cancers $P=0.0000$; endometrial polyps $P=$ 0.0023 ; metastatic tumors $P=0.000579$; ovarian cancers $P=0.0000)$, with the exception of benign epithelial ovarian tumors $(P=0.1834)$. In postmenopausal women, statistically significant differences were observed only in comparisons with groups of patients with oncological diagnoses (endometrial cancers $P=0.0000$; ovarian cancers $P=0.0000$; metastatic tumors $P=0.0049$; other gynecological cancers $P=0.0034)$. No differences were observed in the remaining groups of patients with benign gynecological disorders. In premenopausal BRCA1 mutation carriers, significantly lower serum HE4 levels were observed in comparison to patients with ovarian cancer $(P=0.0000)$, other gynecological cancers $(P=0.0002)$, uterine myomas $(P=0.0021)$, noncancer ovarian cysts $(P=0.0000)$, and adnexitis $(P=0.0035)$. No differences in mean HE4 levels were observed between BRCA1 mutation carriers and patients with endometrial polyps $(P=0.0669)$ or benign epithelial tumors $(P=$ 0.6287 ). No comparisons were made to the group of endometrial cancer patients due to the low number of diagnosed cases in the premenopausal population.

Mean CA125 levels in BRCA1 mutation carriers were statistically different as compared to all study groups with the exception of endometrial polyps. Similarly, to HE4 levels, CA125 levels were the lowest in genetically burdened patients among all the study groups.

Table 2 presents mean serum HE4 and CA125 levels in ovarian cancer patients and other study groups. We demonstrated that serum HE4 and CA125 levels are in most cases statistically higher in ovarian cancer patients as compared to the remaining study groups. Lack of differences was demonstrated only in premenopausal patients with pelvic inflammatory disease (both HE4 and CA125), postmenopausal women diagnosed with endometrial cancer (HE4), and patients with ovarian metastatic tumors (HE4 and CA125 only).

Mean HE4 and CA125 levels were also compared between individual subgroups. Table 3 lists the means, median, and ranges of the measured concentrations. We observed statistically higher serum HE4 levels in vulvar cancer patients (90.62 pmol/L) as compared to endometrial cancer patients $(56.13 \mathrm{pmol} / \mathrm{L}) ; P=0.0106$ as well as statistically higher serum HE4 levels in patients with serous $(720.67 \mathrm{pmol} / \mathrm{L})$ and endometrial $(419.07 \mathrm{pmol} / \mathrm{L})$ ovarian cancers as compared to mucinous type of ovarian cancers $(136.97 \mathrm{pmol} / \mathrm{L})$ with significance levels of $P=0.0070$ and $P=0.0431$, respectively. No differences in HE4 or CA125 levels were observed in the remaining cases.

Figure 1 presents distribution of $\mathrm{HE} 4$ results within the study groups. It is evident that median HE4 concentrations were the highest for ovarian cancers (409.75) followed by endometrial cancers (97.3), other gynecological cancers (58.15), noncancer ovarian cysts (47.45), benign epithelial tumors (47.27), endometriosis (46.5), myomas (44.65), and BRCA1 mutation carriers (38.25). The graph does not include patients with adnexitis (median 54.3) and metastatic tumors (median 209.9) due to the low number of cases. The pattern of serum HE4 levels in pre- and postmenopausal patients is similar, with the highest medians in the ovarian cancer and endometrial cancer groups and the lowest medians in the BRCA1 mutation groups.

ROC curves presented in Figure 2 illustrate the usefulness of HE4 as a diagnostic assay. The relative area under the curve was 0.892 for differentiation of ovarian cancer from 


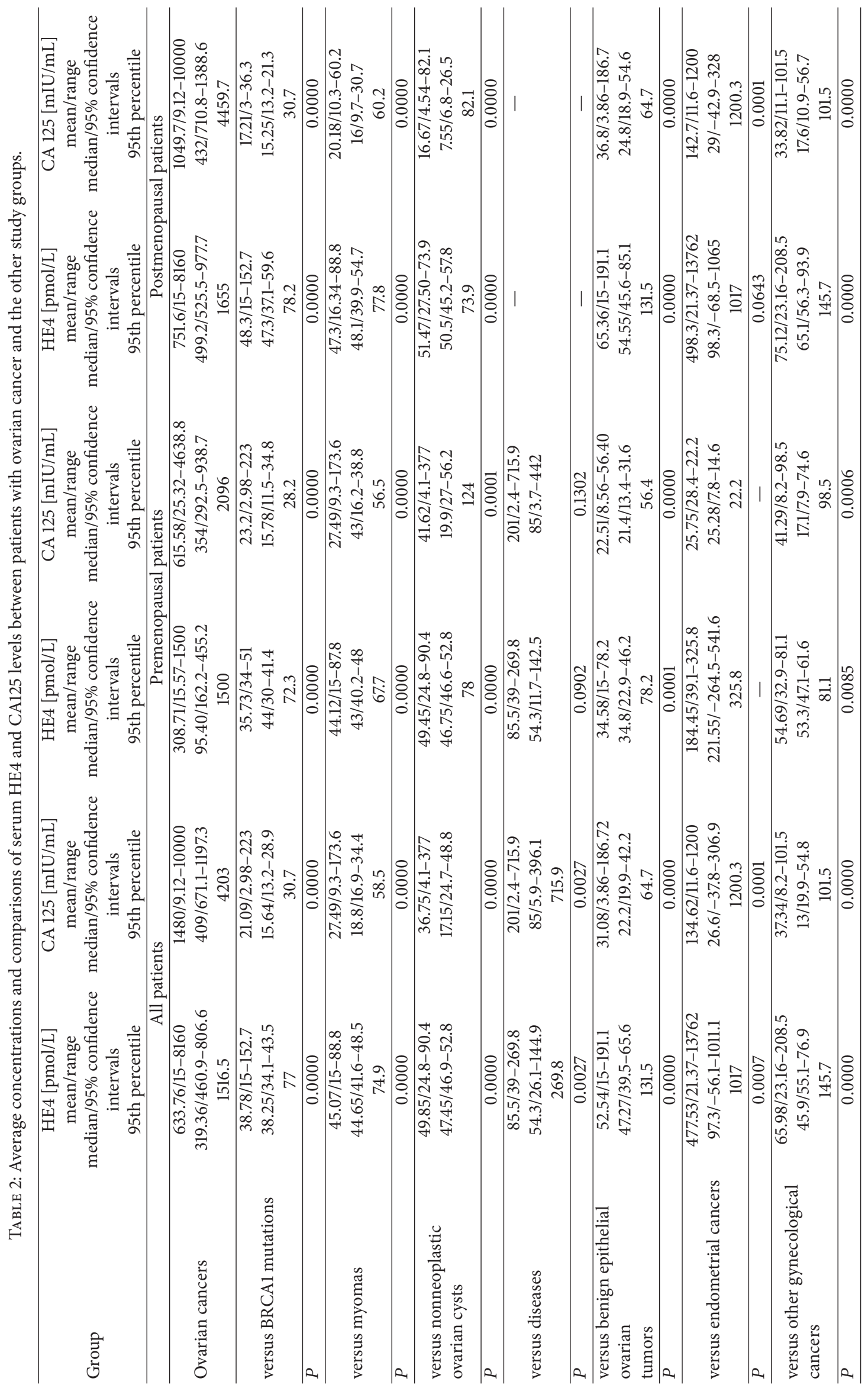




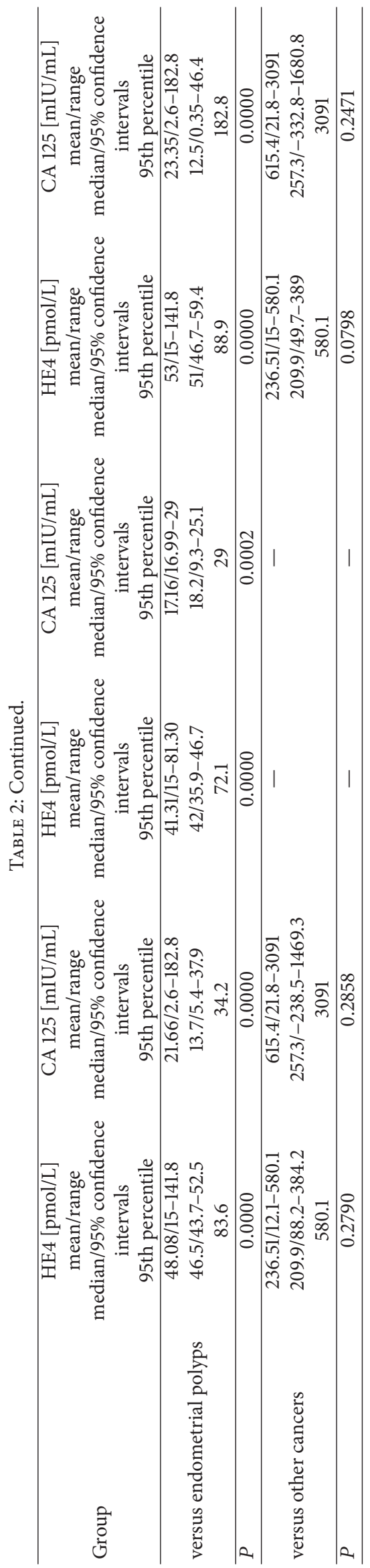




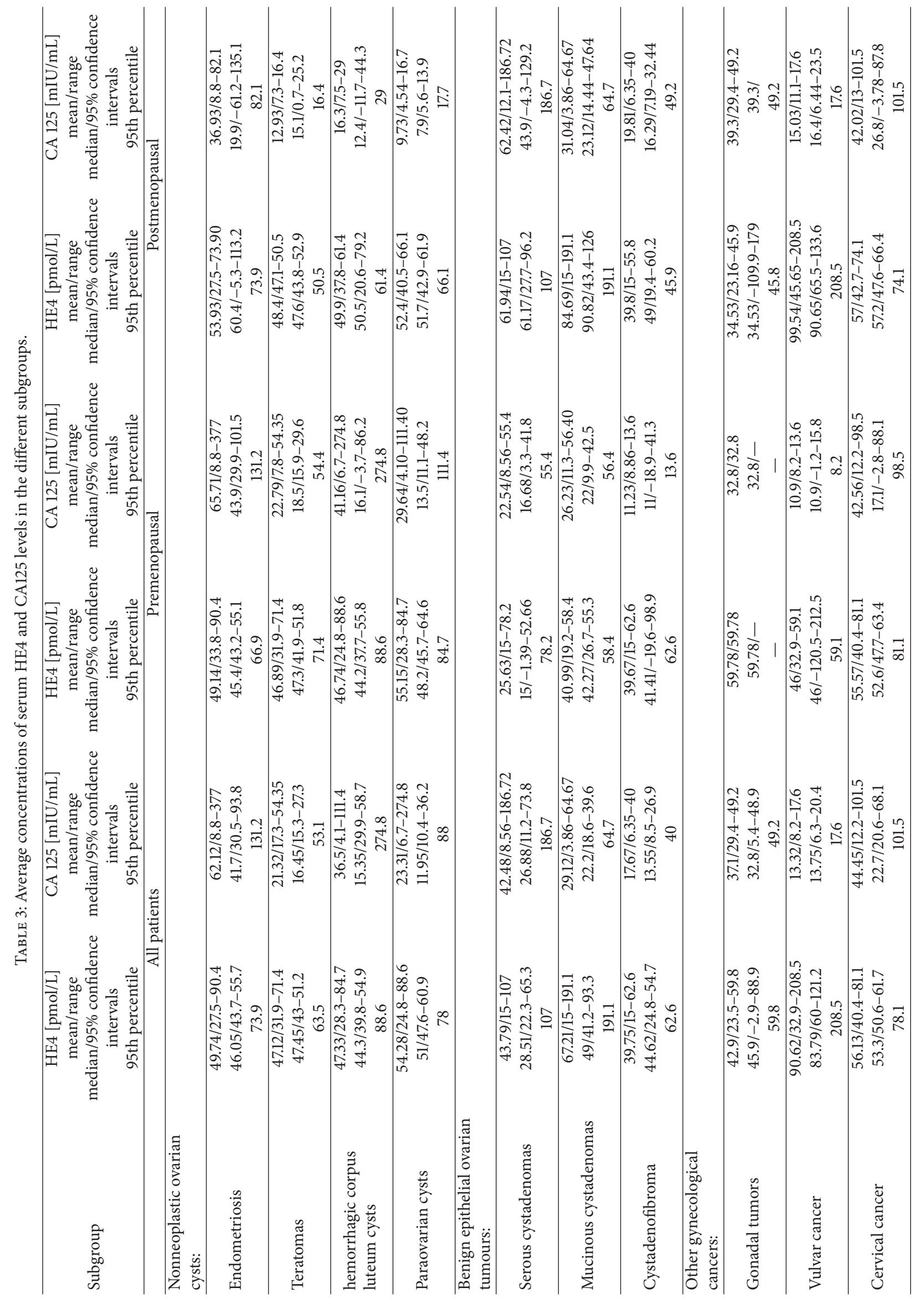




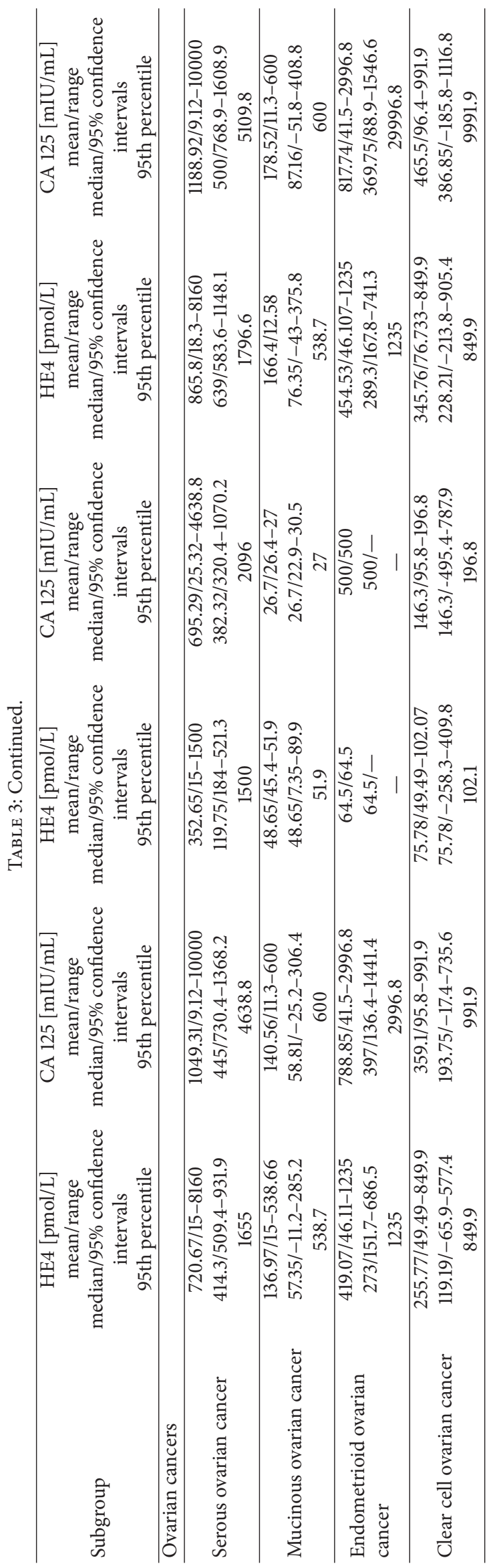



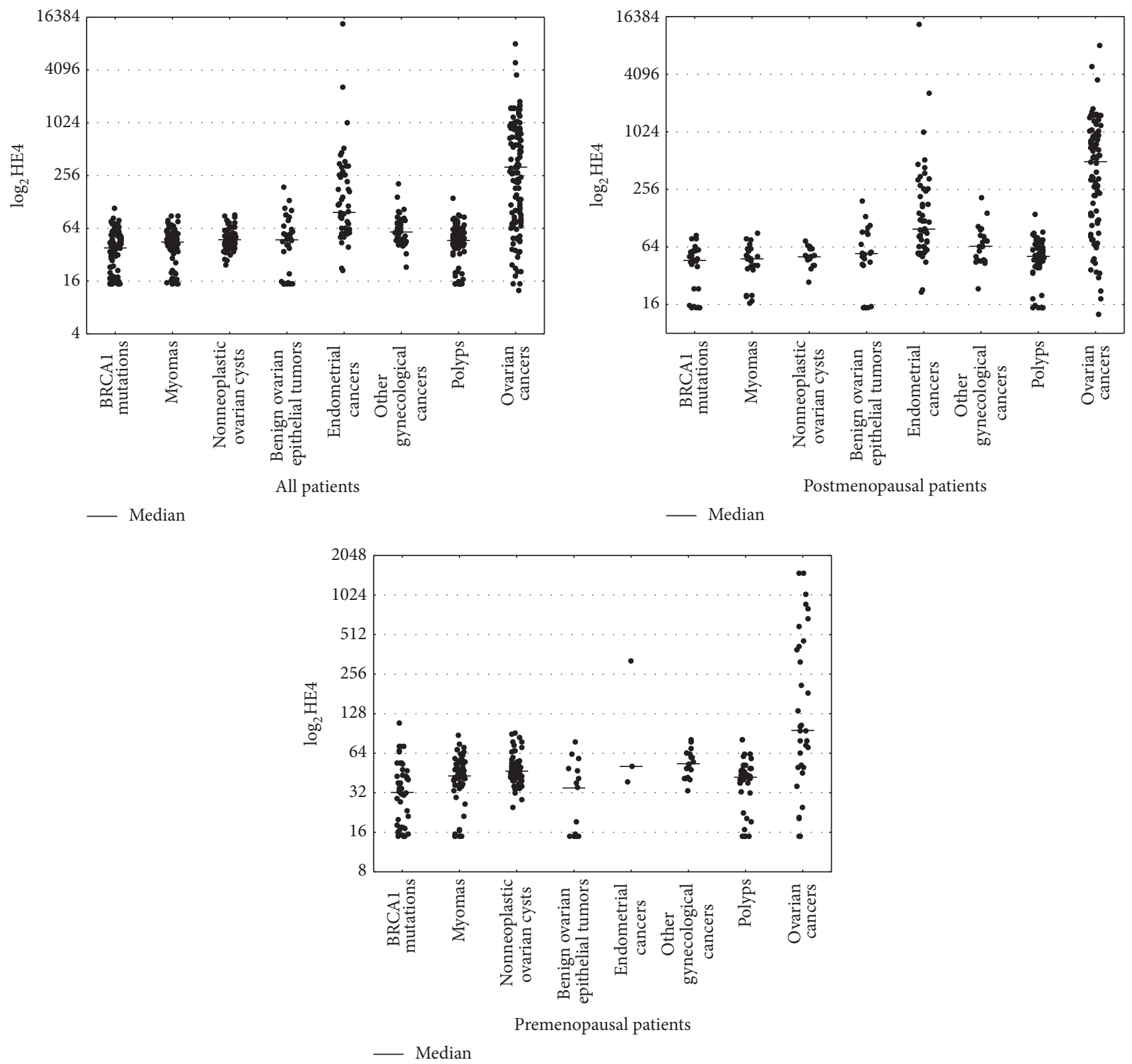

FIGURE 1: Scatterplot of serum HE4 levels by histopathological classifications.

benign nonneoplastic ovarian cysts, 0.894 for differentiation of ovarian cancer from benign epithelial tumors, and 0.865 for differentiation of endometrial cancer from endometrial polyps. The respective ROC AUCs for CA125 were 0.932, 0.936 , and 0.782 . The differences between the ROC AUCs for CA125 and HE4 were statistically significant at $P=0.0148$ for ovarian cancers versus noncancer ovarian cysts, $P=0.0076$ for ovarian cancers versus benign epithelial tumors, and $P=$ 0.0062 for endometrial cancers versus endometrial polyps.

Statistically significant correlations between the serum levels of CA125 and HE4 were observed in the ovarian cancer group ( $r=0.3547, P=0.0001)$, endometrial cancer group $(r=0.7986, P=0.0011)$, and benign epithelial tumor group
( $r=0.3629, P=0.0349$ ). In the remaining groups, no correlations were found between CA125 and HE4 levels.

A detailed analysis of BRCA1 carriers showed ovarian and fallopian tube cancer diagnosed in the postoperative material in 3 out of 83 cases (3.6\%). Patient 1, aged 50, postmenopausal, had reported prophylactic surgery while being asymptomatic. Preoperative markers are CA125: $63.9 \mathrm{U} / \mathrm{mL}$ and HE4: $44.0 \mathrm{pmol} / \mathrm{L}$. Laparoscopic hysterectomy and adnexectomy were performed as a part of prophylactic surgery. Intraoperatively, both ovaries were bilaterally unremarkable; no ascites or peritoneal spread was observed. Postoperative examination revealed a poorly differentiated (G3) serous cancer involving the entire left ovary. Right ovarian tissue 


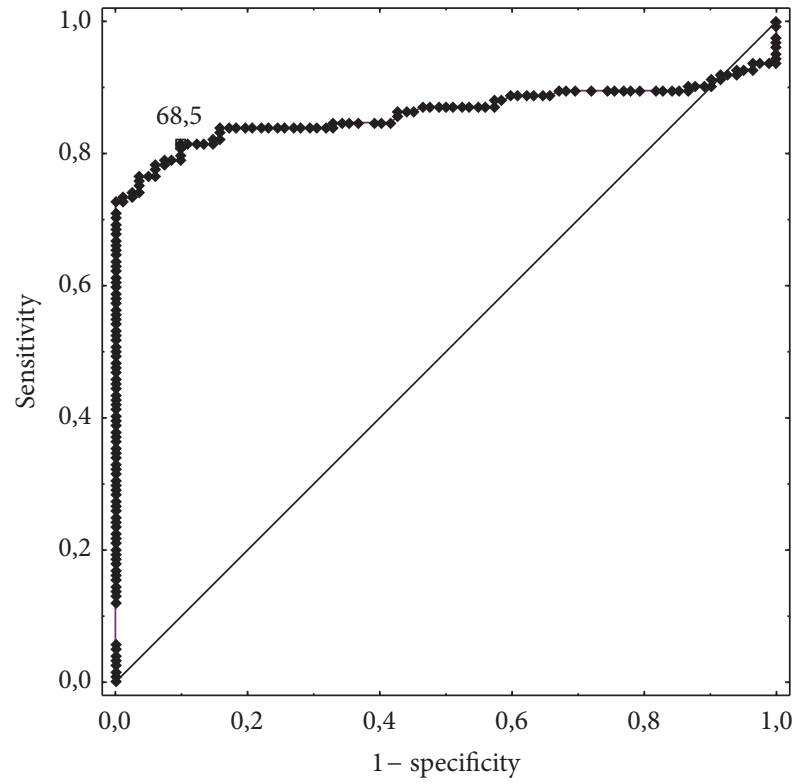

Ovarian cancers versus nonneoplastic ovarian cysts; AUC $=0.892$

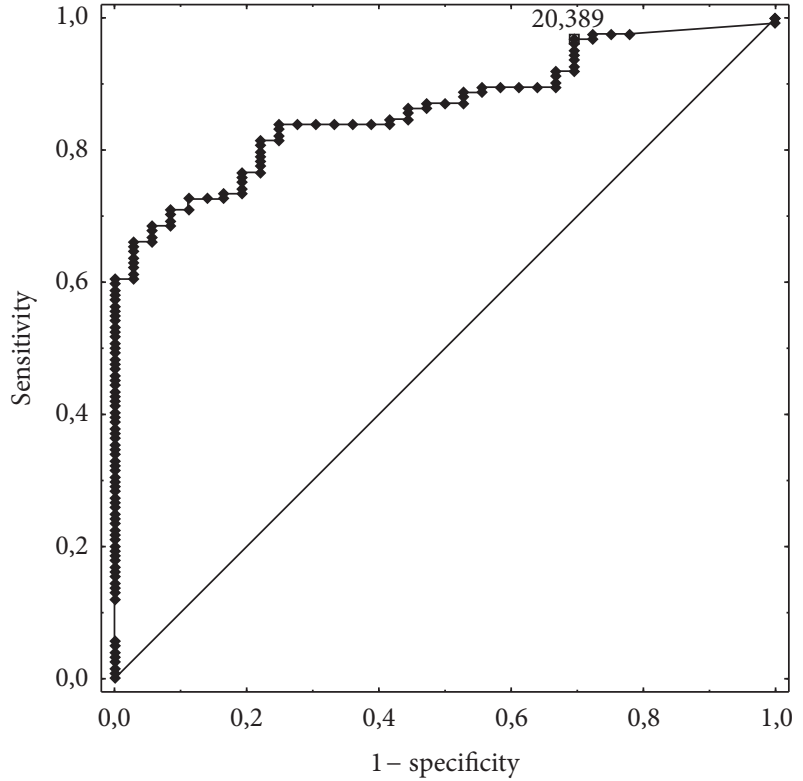

Ovarian cancers versus benign ovarian epithelial tumors; AUC $=0.894$

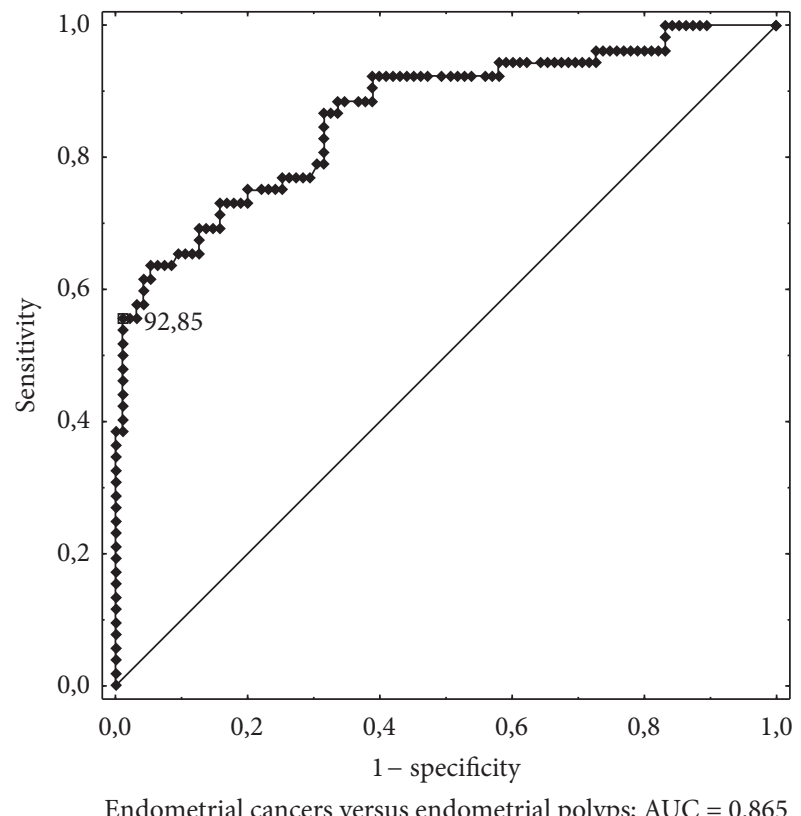

FIgURE 2: Receiver operating curves for HE4 in ovarian and endometrial cancer.

is unremarkable. Another surgery was carried out including the full ovarian cancer procedure. Postoperative material revealed metastases only within para-aortic lymph nodes; patient was classified as FIGO stage III. Patient 2, age 41, clinically asymptomatic, had reported prophylactic surgery. Preoperative markers are CA125: 14.0 U/mL and HE4: $79 \mathrm{pmol} / \mathrm{L}$. Bilateral laparoscopic adnexectomy was carried out with no macroscopic lesions identified within the adnexa. A G2 serous cancer was diagnosed in postoperative material $(20 \%$ of ovary involved). The contralateral ovary and bilateral fallopian tubes were free of tumor infiltration. Another surgery including hysterectomy, adnexectomy, and para-aortic and iliac lymphadenectomy was performed. No cancer cells were identified in any postoperative specimen, confirming the very early clinical stage of the disease (FIGO I). Patient 3, age 39, was also asymptomatic. Preoperative markers are CA125: $39.4 \mathrm{U} / \mathrm{mL}$ and HE4: $32.4 \mathrm{pmol} / \mathrm{L}$. Laparoscopic hysterectomy and adnexectomy were performed; ovaries and fallopian tubes were macroscopically unremarkable. Postoperative histopathological examination revealed G1 cancer of a fallopian tube with massive exfoliation of cancer cells to the tubal lumen with focal infiltration of the perivascular 
parenchyma; features of intraepithelial neoplasm within the other tube. Ovaries were bilaterally unremarkable. Subsequent surgery consisted of hysterectomy and omentectomy as well as para-aortic and iliac lymphadenectomy. No cancer cells were identified in the postoperative material. The final diagnosis was G1 fallopian tube cancer, FIGO stage I.

Twenty-four (30\%) of 80 patients with BRCA1 mutations had been previously diagnosed and treated for breast cancer. No patient had an active disease upon being qualified for the study. All patients were in remission. Mean HE4 level in this subgroup was $35.9 \mathrm{pmol} / \mathrm{L}$. Over several years of followup, we observed 4 additional breast cancer cases occurring within the period of 6 months to 3 years after the prophylactic surgery. One patient suffered a breast cancer relapse 6 months after surgery. One patient developed primary peritoneal cancer 8 months after the prophylactic surgery, accounting for $1.25 \%$ of the entire study group. At the time of prophylactic surgery, the HE4 level was $15 \mathrm{pmol} / \mathrm{L}$. At the time of diagnosis of primary peritoneal cancer, it was $454.3 \mathrm{pmol} / \mathrm{L}$.

\section{Discussion}

The studies aimed at the development of an ovarian cancer screening test have been ongoing for several decades. After thousands of women were studied none of the methods proposed to date could meet the criteria of a screening test. Methods attempted to date were inefficient in reducing the mortality due to ovarian cancer or in increasing the percentage of women in whom the disease is diagnosed at an early stage. In addition, a high percentage of false positive results leads to unnecessary surgeries which are associated with complications and stress [18]. Only the group of patients genetically predisposed to the ovarian cancer benefits from prophylactic screening which should lead to prophylactic salpingooophorectomy at an appropriate age $[16,17]$. The assay that has been used most commonly in the screening of patients genetically predisposed to ovarian cancer is determination of serum CA125 levels and annual transvaginal ultrasound scans. Recent studies conducted in a group of patients at high risk of ovarian cancer showed that the risk of ovarian cancer algorithm (ROCA), consisting of serial determinations of CA125 levels, individualization of these levels for each patient, and stratification of patients into ovarian cancer risk strata determining further management may be of high clinical usefulness [19].

The researches expect new possibilities for the ovarian cancer screening to be offered by the novel cancer marker, HE4, used to date along with CA125 mainly in the diagnostics of pathological developments within the adnexa [1-10]. To date, only a few articles assessing the levels of this marker in a group of females at high risk of ovarian cancer were studied [20-22]. Shah et al. [20] demonstrated that a mean HE4 level was not significantly different in healthy females at high and medium risk of ovarian cancer, although the mean values were somewhat lower in the high-risk group. The CA125 levels were statistically lower in high-risk subjects. According to the authors, the high-risk group consisted of patients with a positive family history (at least two ovarian or breast cancers in first- or second-degree relatives), BRCA1 carriers, and Ashkenazi Jews with a positive family history (one ovarian or breast cancer in a first-degree relative or two cancers in second-degree relatives). When assessing the diagnostic usefulness of CA125 and HE4 levels in ovarian cancer, the researchers determined that the area under the ROC curve was not statistically different when the control group consisted of patients with medium (CA125: 0.939, HE4: 0.928) or high (CA125: 0.939, HE4: 0.931) risk of ovarian cancer. Anderson et al. [21] evaluated the potential for predicting ovarian cancer using a symptom index, CA125, and HE4 as a multimodality, multistage screening program. When analyzing different combinations of these three parameters, the researchers came to a conclusion that the presence of 2 of 3 analyzed parameters as the first line of ovarian cancer is characterized by specificity of ca. $98.5 \%$. They also suggested that inclusion of transvaginal ultrasound scan as the second line of screening might increase the specificity and PPV to the acceptable level. Patients at high risk of ovarian cancer were also the subjects of the study conducted by Urban et al. [22]. The authors assumed that HE4 might be useful in ovarian cancer screening, as the reference standards presented to date are contradictory. In their publication they decided to determine the reference standards of HE4 for patients with BRCA 1 mutations in age above 25 and for patients with family history of breast or ovarian cancer in age above 35 . Serial analyses were conducted on individual HE4 and CA125 levels depending on the age, race, hormone replacement therapy status, contraception status, smoking status, and history of oophorectomy or tubal ligation. The study results showed that HE4 was lower in black females and higher in smokers and markedly increased with age, particularly after the age of 55 , which should be taken into account when deciding upon screening tests based on HE4 determinations. In our studies, mean HE4 levels were assessed in a group of patients at the highest risk of ovarian cancer, that is, in patients diagnosed with BRCA1 gene mutation. According to current estimates, $5-15 \%$ of all ovarian cancers are associated with germinal mutations, with $90-95 \%$ of these consisting of mutations within BRCA1/2 genes [23]. The risk of ovarian cancer in the overall population is about $1.6 \%$ while it reaches up to $60 \%$ in BRCA1 carriers [23]. As shown by our analysis of 80 BRCA1 carriers, mean levels of the marker in the study populations were significantly lower than in the group of females with benign gynecological disorders (functional and nonneoplastic cysts, uterine myomas, and endometrial polyps) in the whole examined groups and premenopausal patients. There were no significant differences in the mean age of patients in the study groups, which is particularly important as HE4 levels are age-dependent. In our study we do not analyzed group of healthy women. However, comparing our results with those of healthy women cited by Roche in the summary of product characteristics (http://www.cobas.com/content/dam/cobas_com/pdf/product/ Elecsys HE4 Human Epididymal Protein 4/HE4 fact sheet.pdf) also shows a trend towards lower values of serum HE4 levels in carriers of BRCA 1 compared with healthy women. To make this analysis we divided our patients with the same age range as quoted by Roche study and compared median 
values. The results were as follows: in the under 40 years of age median value of HE4 in carriers was $41 \mathrm{pmol} / \mathrm{L}$ and $42 \mathrm{pmol} / \mathrm{L}$, in healthy women, between $40-49$ years of age, respectively, it was $31.1 \mathrm{pmol} / \mathrm{L}$ and $44.3 \mathrm{pmol} / \mathrm{L}$, in the range of 50-59 years, it was $44.7 \mathrm{pmol} / \mathrm{L}$ and $47.9 \mathrm{pmol} / \mathrm{L}$, and in the range 60-69 years in carriers of BRCA 1 and healthy women values were identical $(55 \mathrm{pmol} / \mathrm{L})$. Of course, due to the lack of source data for healthy women statistical comparisons were not performed which is a defect of this analysis. None of the three papers cited above [21, 22] assessed HE4 levels in a group of BRCA1 gene mutation carriers only. BRCA mutation carriers were included in the high-risk groups along with females with family history but no genetic mutations. Therefore, this report is the first to present the values of HE4 levels in a group of female patients with BRCA1 gene mutation. The values in high-risk patients as reported by Urban et al. [22] were higher than those observed in our study. In the group of premenopausal women with the mean age of 43 years, the mean HE4 level measured in our study was $35.7 \mathrm{pmol} / \mathrm{L}$ as compared to $27.7 \mathrm{pmol} / \mathrm{L}$ reported by the aforementioned authors; the respective values in postmenopausal women were $48.3 \mathrm{pmol} / \mathrm{L}$ in our study and $31.3 \mathrm{pmol} / \mathrm{L}$ in the study by Urban et al. [22]. However, one should take note of different laboratory methods being used in Roche versus Abbott studies. In the study by Urban et al. [22], patients with BRCA1/2 mutations accounted for $17.7 \%$ (138 cases) of the population included in the analysis. It appears that when deciding upon the future use of HE4 determination in ovarian cancer screening, the low values measured in patients with germinal BRCA1 mutation should be taken into account, which obviously requires further research in a significantly larger population of females.

Occult ovarian cancer was diagnosed by histopathological examination in 3 out of 83 (3.6\%) women undergoing surgery. In either of these three cases, the ovaries were macroscopically unremarkable. All 83 surgeries were carried out by means of laparoscopic technique. The surgeries included bilateral adnexectomies in most cases and subtotal hysterectomies in selected cases when requested by the patient or when they are due to uterine myomas. The incidence of occult ovarian/fallopian tube cancers in patients having undergone prophylactic surgeries for BRCAl gene mutation is varied [24-26] and ranges from $1.9 \%$ [25] to $16.2 \%$ [26]. Similarly at our center, most surgeries are carried out by laparoscopic technique which appears to be safe and worth recommendation $[27,28]$. The wide distribution of results regarding the diagnosis of latent ovarian cancer in BRCA1 gene mutation carrier is most probably due to different sizes of the study samples and average ages of patients when undergoing the prophylactic surgery (the populations of patients in cited studies ranged from 37 [26] to 374 [29] females). Regardless of the cited incidence of occult ovarian cancer, particular care is recommended during prophylactic surgeries consisting of delicate removal of ovaries from the abdominal cavity, preferably using endobags, so as to avoid potential cancer spread as well as in appropriate preparation of the team of pathologists who should be aware of the very small size of possible neoplastic foci within the ovaries of patients at high risk of cancer. Of particular note is the fact that each of the 3 patients with latent ovarian cancer in our study presented with discrete elevation of one of the two markers. Therefore, one might consider a management approach involving each patient qualified for prophylactic surgery being subjected to HE 4 and CA125 determination; in case of elevation of one of the markers, the range of diagnostic procedures should be widened, intraoperative examination included.

HE4 is a relatively novel tumor marker used in the diagnostics of pathological lesions within the adnexa. Its sensitivity is very similar to that of CA125 while its specificity is much higher $[2,8,10,14,30]$. Despite reports that differ in the assessment of diagnostic usefulness of HE4, suggesting either CA125 $[8,30]$ or HE4 $[2,10,14]$ as the more useful marker, HE4 has already become an established marker in the diagnostics of ovarian cancer. Our results confirm the high diagnostic value of HE4 despite the fact that better results were obtained for CA125. Both markers based on AUC values for the ROC curves meet the criteria of a good diagnostic test and similar results have been published by other authors $[2,10]$, postulating simultaneous use of both markers in the diagnostics of ovarian cancer, for example, with employing the ROMA algorithm.

No cancer marker of potential importance in preoperative diagnostics has been found to date for endometrial cancer. Based on the research conducted in recent years, it appears that this vacancy may be filled by HE4 [31-37]. In 2013, Jiang et al. [37] demonstrated very high tissue expression of HE4 in endometrial cancer tissues. This was confirmed by Li et al. [31] who additionally demonstrated that high values of the marker's levels are correlated with tumor size, clinical stage, and depth of myometrial involvement while tissue overexpression has an influence on the progression of cancer. The authors reported the clinical usefulness of HE4 in both early [38] and advanced stages of endometrial cancer [33]. The results of our studies are consistent with those obtained by other authors and confirm that endometrial cancer is accompanied by elevated HE4 levels. The diagnostic value of HE4 is confirmed by high AUC value (0.865) calculated for endometrial cancer relative to endometrial polyps. The value was statistically significantly higher as compared to the ROC-AUC value for CA125 (0.651). In the studies by Moore et al. [38], ROC-AUC in endometrial cancer was 0.671 for CA125 and 0.787 for HE4 and $P=0.0007$. The authors assessed endometrial cancers of all stages as compared to healthy volunteers. Similar results were presented by Saarelainen et al. [33], with ROC-AUC amounting to 0.76 for HE4 and 0.65 for CA125. How can we therefore make use of the measurements of serum HE4 levels in endometrial cancer? While preoperative diagnostics putting forth the suspicion of endometrial cancer is relatively straightforward due to typical symptoms, risk factors, and transvaginal ultrasound results, a method helpful in making decisions regarding the surgical treatment is still being sought for. Iliac and para-aortic lymphadenectomy is recommended in patients with high-risk factors (G3, tumor size of more than $2 \mathrm{~cm}$, involvement of vascular spaces, and deep involvement of myometrium). Unfortunately, data on these risk factors are usually unavailable at the moment of decision regarding the surgical treatment. The statistically 
significant high concentrations of $\mathrm{HE} 4$ in endometrial cancer and as observed in our study as well as the correlation of these concentrations with high-risk factors reported by other authors $[31,33,38]$ should warrant the conduct of further prospective studies.

\section{Conclusion}

Patients with BRCA1 gene mutations are characterized by relatively low serum HE4 levels. The trend is particularly evident in younger, premenopausal females. Determination of CA125 and HE4 in every patient carrying the BRCA1 gene mutation and undergoing prophylactic surgery appears justifiable. Even the slightest elevation in HE4 or CA 125 levels in these patients should significantly increase oncological alertness. The HE4 marker is a valuable tool not only in differentiation of malignant and benign lesions within the adnexa, but also in differentiation of malignant and benign disorders of the uterine endometrium.

\author{
Abbreviations \\ ROMA: Risk of Malignancy Algorithm \\ HE4: Human epididymal protein 4 \\ CA 125: Cancer antigen 125 \\ FSH: Follitropin \\ ROC: Receiver operating characteristic \\ AUC: Area under curve \\ ROCA: Risk of ovarian cancer antigen \\ PPV: $\quad$ Positive predictive value \\ NPV: $\quad$ Negative predictive value.
}

\section{Ethical Approval}

Ethics permission for the study was approved by the Bioethics Committee of Pomeranian Medical University (protocol no. KB-0012/58/11). All patients gave written consent.

\section{Disclosure}

All authors are from Department of Gynecological Surgery and Gynecological Oncology of Adults and Adolescents, Pomeranian Medical University of Szczecin, Poland.

\section{Competing Interests}

The authors declare that they have no competing interests.

\section{Authors' Contributions}

Anita Chudecka-Głaz has made substantial contributions to conception and design, planned the experiments, collected data, performed analysis (statistics) and interpretation of the results, reviewed the literature, and wrote the manuscript. Aneta Cymbaluk-Płoska has contributed in collecting data. Janusz Menkiszak has given the approval for the final version to be published. Aleksandra Strojna has contributed in collecting data.

\section{Acknowledgments}

Thanks are due to the staff of the Central Laboratory SPSK No. 2 Pomeranian Medical University in Szczecin for carrying out the assays of tumor markers.

\section{References}

[1] I. Hellström, J. Raycraft, M. Hayden-Ledbetter et al., "The HE4 (WFDC2) protein is a biomarker for ovarian carcinoma," Cancer Research, vol. 63, no. 13, pp. 3695-3700, 2003.

[2] Y. Park, J.-H. Lee, D. J. Hong, E. Y. Lee, and H.-S. Kim, "Diagnostic performances of HE4 and CA125 for the detection of ovarian cancer from patients with various gynecologic and non-gynecologic diseases," Clinical Biochemistry, vol. 44, no. 1011, pp. 884-888, 2011.

[3] Z. Novotny, J. Presl, R. Kucera et al., "HE4 and ROMA index in Czech postmenopausal women," Anticancer Research, vol. 32, no. 9, pp. 4137-4140, 2012.

[4] C. Anton, F. M. Carvalho, E. I. Oliveira, G. A. R. Maciel, E. C. Baracat, and J. P. Carvalho, "A comparison of CA125, HE4, risk ovarian malignancy algorithm (ROMA), and risk malignancy index (RMI) for the classification of ovarian masses," Clinics, vol. 67, no. 5, pp. 437-441, 2012.

[5] R. G. Moore, M. C. Miller, M. M. Steinhoff et al., "Serum HE4 levels are less frequently elevated than CA125 in women with benign gynecologic disorders," American Journal of Obstetrics \& Gynecology, vol. 206, no. 4, pp. 351.el-351.e8, 2012.

[6] R. G. Moore, D. S. McMeekin, A. K. Brown et al., "A novel multiple marker bioassay utilizing HE4 and CA125 for the prediction of ovarian cancer in patients with a pelvic mass," Gynecologic Oncology, vol. 112, no. 1, pp. 40-46, 2009.

[7] R. Molina, J. M. Escudero, J. M. Augé et al., "HE4 a novel tumour marker for ovarian cancer: comparison with CA 125 and ROMA algorithm in patients with gynaecological diseases," Tumour Biology, vol. 32, no. 6, pp. 1087-1095, 2011.

[8] H. Zheng and Y. Gao, "Serum HE4 as a useful biomarker in discriminating ovarian cancer from benign pelvic disease," International Journal of Gynecological Cancer, vol. 22, no. 6, pp. 1000-1005, 2012.

[9] R. G. Moore, A. K. Brown, M. C. Miller et al., "The use of multiple novel tumor biomarkers for the detection of ovarian carcinoma in patients with a pelvic mass," Gynecologic Oncology, vol. 108, no. 2, pp. 402-408, 2008.

[10] K. Partheen, B. Kristjansdottir, and K. Sundfeldt, "Evaluation of ovarian cancer biomarkers HE4 and CA-125 in women presenting with a suspicious cystic ovarian mass," Journal of Gynecologic Oncology, vol. 22, no. 4, pp. 244-252, 2011.

[11] H. Fujiwara, M. Suzuki, N. Takeshima et al., "Evaluation of human epididymis protein 4 (HE4) and Risk of Ovarian Malignancy Algorithm (ROMA) as diagnostic tools of type I and type II epithelial ovarian cancer in Japanese women," Tumor Biology, vol. 36, no. 2, pp. 1045-1053, 2015.

[12] R. G. Moore, M. Jabre-Raughley, A. K. Brown et al., "Comparison of a novel multiple marker assay vs the Risk of Malignancy Index for the prediction of epithelial ovarian cancer in patients with a pelvic mass," American Journal of Obstetrics and Gynecology, vol. 203, no. 3, pp. 228.e1-228.e6, 2010.

[13] E. I. Braicu, R. Chekerov, R. Richter et al., "HE4 expression in plasma correlates with surgical outcome and overall survival in patients with first ovarian cancer relapse," Annals of Surgical Oncology, vol. 21, no. 3, pp. 955-962, 2014. 
[14] E. Bandiera, C. Romani, C. Specchia et al., "Serum human epididymis protein 4 and risk for ovarian malignancy algorithm as new diagnostic and prognostic tools for epithelial ovarian cancer management," Cancer Epidemiology Biomarkers and Prevention, vol. 20, no. 12, pp. 2496-2506, 2011.

[15] S. S. Buys, E. Partridge, A. Black et al., "Effect of screening on ovarian cancer mortality: the Prostate, Lung, Colorectal and Ovarian (PLCO) cancer screening randomized controlled trial," The Journal of the American Medical Association, vol. 305, no. 22, pp. 2295-2302, 2011.

[16] H. D. Nelson, R. Fu, K. Goddard et al., Risk Assessment, Genetic Counselling, and Genetic Testing for BRCA-Related Cancer: Systematic Review to Update the U.S. Preventive Services Task Force Recommendation, Agency for Healthcare Research and Quality, Rockville, Md, USA, 2013.

[17] A. P. M. Finch, J. Lubinski, P. Møller et al., "Impact of oophorectomy on cancer incidence and mortality in women with a BRCA1 or BRCA2 mutation," Journal of Clinical Oncology, vol. 32, no. 15, pp. 1547-1553, 2014.

[18] C. J. Reade, J. J. Riva, J. W. Busse, C. H. Goldsmith, and L. Elit, "Risks and benefits of screening asymptomatic women for ovarian cancer: a systematic review and meta-analysis," Gynecologic Oncology, vol. 130, no. 3, pp. 674-681, 2013.

[19] M. E. Sherman, M. Piedmonte, P. L. Mai et al., "Pathologic findings at risk-reducing salpingo-oophorectomy: primary results from Gynecologic Oncology Group trial GOG-0199," Journal of Clinical Oncology, vol. 32, no. 29, pp. 3275-3283, 2014.

[20] C. A. Shah, K. A. Lowe, P. Paley et al., "Influence of ovarian cancer risk status on the diagnostic performance of the serum biomarkers mesothelin, HE4, and CA125," Cancer Epidemiology Biomarkers and Prevention, vol. 18, no. 5, pp. 1365-1372, 2009.

[21] G. L. Anderson, M. McIntosh, L. Wu et al., "Assessing lead time of selected ovarian cancer biomarkers: a nested case-control study," Journal of the National Cancer Institute, vol. 102, no. 1, pp. 26-38, 2010.

[22] N. Urban, J. Thorpe, B. Y. Karlan et al., "Interpretation of single and serial measures of HE4 and CA 125 in asymptomatic women at high risk for ovarian cancer," Cancer Epidemiology, Biomarkers \& Prevention, vol. 21, no. 11, pp. 2067-2094, 2012.

[23] S. Zhang, R. Royer, S. Li et al., "Frequencies of BRCA1 and BRCA2 mutations among 1,342 unselected patients with invasive ovarian cancer," Gynecologic Oncology, vol. 121, no. 2, pp. 353-357, 2011.

[24] R. I. Olivier, M. van Beurden, M. A. C. Lubsen et al., "Clinical outcome of prophylactic oophorectomy in BRCA1/BRCA2 mutation carriers and events during follow-up," British Journal of Cancer, vol. 90, no. 8, pp. 1492-1497, 2004.

[25] D. G. R. Evans, R. Clayton, P. Donnai, A. Shenton, and F. Lalloo, "Risk-reducing surgery for ovarian cancer: outcomes in 300 surgeries suggest a low peritoneal primary risk," European Journal of Human Genetics, vol. 17, no. 11, pp. 1381-1385, 2009.

[26] M. I. Carcangiu, B. Peissel, B. Pasini, G. Spatti, P. Radice, and S. Monoukian, "Incidental carcinomas in prophylactic specimens in BRCA 1 and BRCA 2 germ-line mutations carriers, with emphasis on fallopian tube lesions: report of 6 cases an review of the literature," The American Journal of Surgical Pathology, vol. 30, no. 10, pp. 1222-1230, 2006.

[27] A. Synowiec, G. Wcisło, L. Bodnar, and C. Szczylik, "Surgical treatment in ovarian cancer prevention in carriers of the BRCA1/BRCA2 mutation," Ginekologia Polska, vol. 83, no. 1, pp. 51-56, 2012.
[28] K. Haldar and R. Crawford, "Risk-reducing salpingooophorectomy for BRCA mutation carriers," Maturitas, vol. 67, no. 3, p. 290, 2010.

[29] A. Finch, M. Beiner, J. Lubiński et al., "Salpingo-oophorectomy and the risk of ovarian, fallopian tube and peritoneal cancers in women with BRCA1 or BRCA2 mutation," The Journal of the American Medical Association, vol. 296, no. 2, pp. 185-192, 2006.

[30] G. Ruggeri, E. Bandiera, L. Zanotti et al., "HE4 and epithelial ovarian cancer: comparison and clinical evaluation of two immunoassays and a combination algorithm," Clinica Chimica Acta, vol. 412, no. 15-16, pp. 1447-1453, 2011.

[31] J. Li, H. Chen, A. Mariani et al., "HE4 (WFDC2) promotes tumor growth in endometrial cancer cell lines," International Journal of Molecular Sciences, vol. 14, no. 3, pp. 6026-6043, 2013.

[32] B. Omer, S. Genc, O. Takmaz et al., "The diagnostic role of human epididymis protein 4 and serum amyloid-A in earlystage endometrial cancer patients," Tumor Biology, vol. 34, no. 5, pp. 2645-2650, 2013.

[33] S. K. Saarelainen, N. Peltonen, T. Lehtimäki, A. Perheentupa, M. H. Vuento, and J. U. Mäenpää, "Predictive value of serum human epididymis protein 4 and cancer antigen 125 concentrations in endometrial carcinoma," American Journal of Obstetrics and Gynecology, vol. 209, no. 2, pp. 142.el-142.e6, 2013.

[34] Z. Yang, C. Wei, Z. Luo, and L. Li, "Clinical value of serum human epididymis protein 4 assay in the diagnosis of ovarian cancer: a meta-analysis," OncoTargets and Therapy, vol. 6, pp. 957-966, 2013.

[35] D. J. Brennan, A. Hackethal, A. M. Metcalf et al., "Serum HE4 as a prognostic marker in endometrial cancer-a population based study," Gynecologic Oncology, vol. 132, no. 1, pp. 159-165, 2014.

[36] A. R. Simmons, K. Baggerly, and R. C. Bast Jr., "The emerging role of HE4 in the evaluation of epithelial ovarian and endometrial carcinomas," ONCOLOGY (United States), vol. 27, no. 6, pp. 548-556, 2013

[37] S.-W. Jiang, H. Chen, S. Dowdy et al., "HE4 transcription- and splice variants-specific expression in endometrial cancer and correlation with patient survival," International Journal of Molecular Sciences, vol. 14, no. 11, pp. 22655-22677, 2013.

[38] R. G. Moore, A. K. Brown, M. C. Miller et al., "Utility of a novel serum tumor biomarker HE4 in patients with endometrioid adenocarcinoma of the uterus," Gynecologic Oncology, vol. 110, no. 2, pp. 196-201, 2008. 


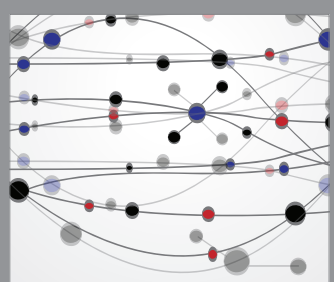

The Scientific World Journal
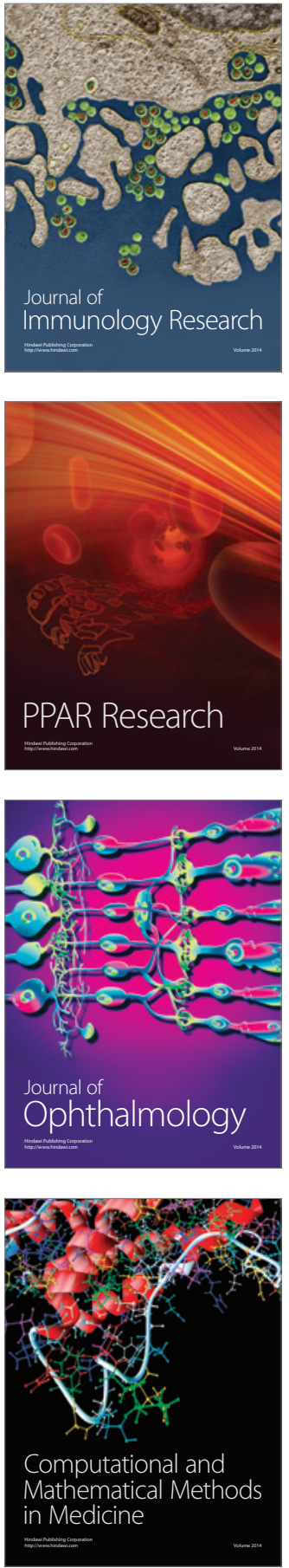

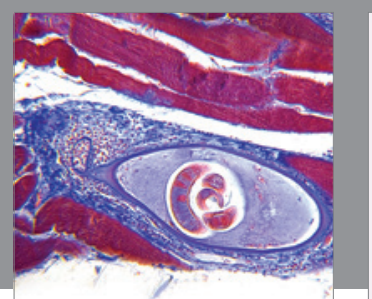

Gastroenterology Research and Practice
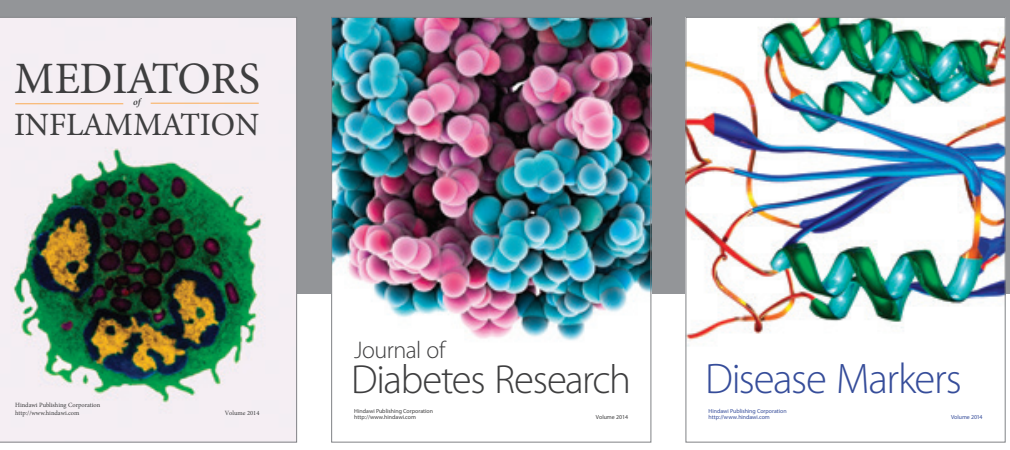

Disease Markers

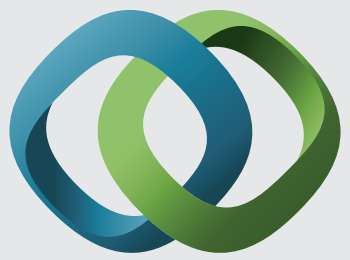

\section{Hindawi}

Submit your manuscripts at

https://www.hindawi.com
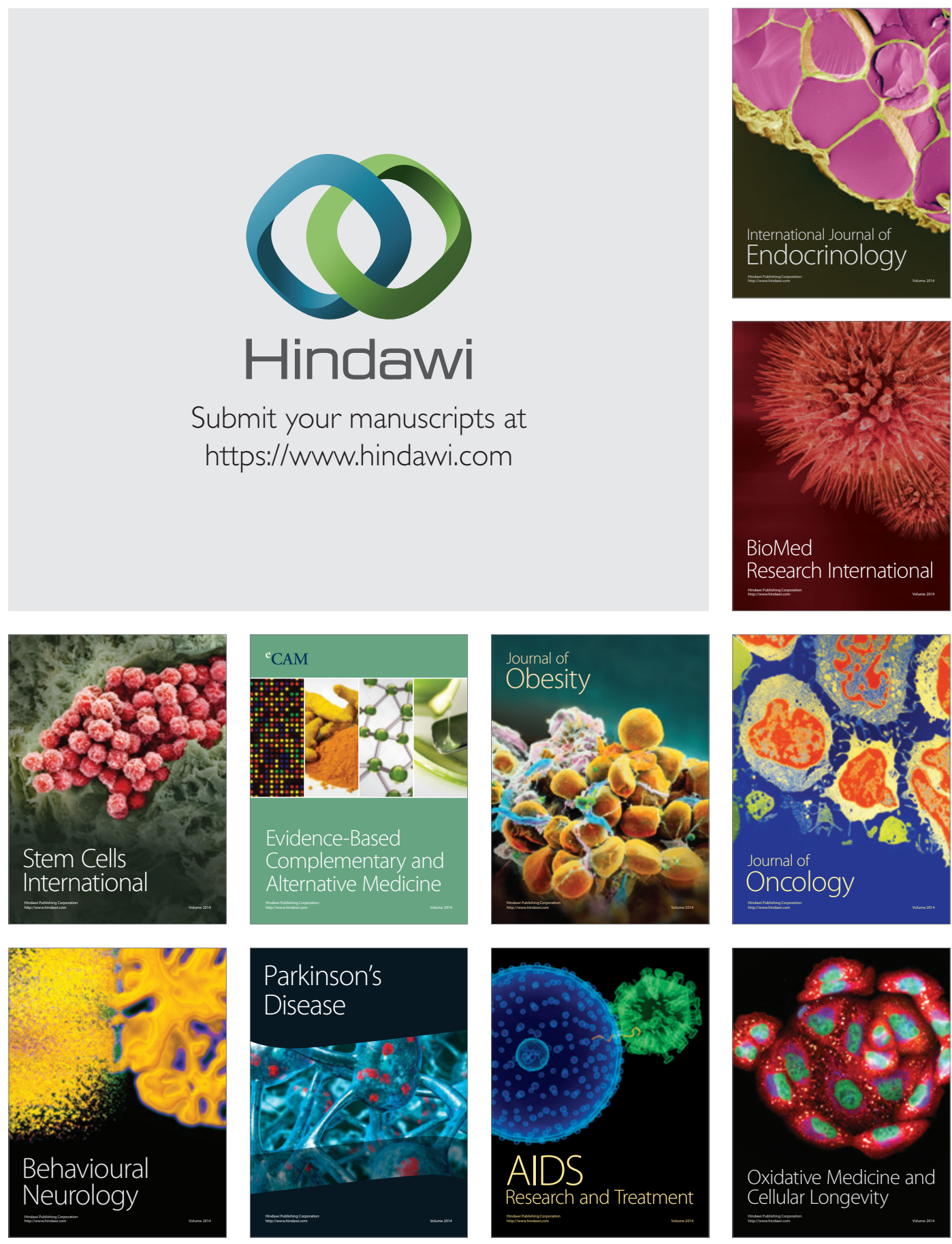